\title{
Design, control, and characterisation of switchable radiative cooling
}

\section{Angus Gentle, Matthew Tai, Simon White, Matthew Arnold, Michael Cortie, et al.}

Angus Gentle, Matthew Tai, Simon White, Matthew Arnold, Michael Cortie, Geoff Smith, "Design, control, and characterisation of switchable radiative cooling," Proc. SPIE 10759, New Concepts in Solar and Thermal Radiation Conversion and Reliability, 107590L (17 September 2018); doi: $10.1117 / 12.2323877$

EDIE Event: SPIE Optical Engineering + Applications, 2018, San Diego, California, United States 


\title{
Design, control, and characterisation of switchable radiative cooling.
}

\author{
Angus Gentle*, Matthew Tai, Simon White, Matthew Arnold, Michael Cortie, Geoff Smith. \\ School of Mathematical and Physical Science, University of Technology Sydney, \\ PO Box 123, Broadway, NSW, 2007, Australia
}

\begin{abstract}
Here we present our recent developments in temperature dependent ellipsometry, FTIR and emittance measurements of flat and structured vanadium dioxide (VO2) surfaces allowing significant control of switchable radiative cooling beyond that attainable via traditional VO2 surfaces. VO2 undergoes a metal-insulator transition at a critical temperature of $68^{\circ} \mathrm{C}$; previous work has investigated tuning of this critical temperature over a wide range of temperatures. Here we exploit the shift in optical properties to produce surfaces with various emittance temperature profiles that modulate the thermal radiative transfer to/from a surface.

Designing surfaces with different temperature emittance profiles requires accurate optical/thermal characterisation of materials. VO2 is produced by sputtering of vanadium followed by post deposition annealing in a 0.1 Torr to 0.3 Torr Air atmosphere at $450^{\circ} \mathrm{C}$ to $550^{\circ} \mathrm{C}$, in-situ optical monitoring allows for accurate termination of the annealing process once the desired optical response is achieved.
\end{abstract}

Keywords: Radiative Cooling, Thermal Emission, vanadium dioxide, vo2, emissivity

\section{INTRODUCTION}

Recent advancements in day-time sub-ambient cooling have led to many groups chasing materials that allow low temperatures to be attained in full sunlight, which is typically achieved by the very high solar reflectances, rather than optimisation of the radiative aspects of the cooling surface. Here we will concentrate on broader aspects of control of radiative cooling, but the measurement techniques discussed are applicable to various radiative cooling applications.

The application of switchable optical properties has been a growing area of research, with materials such as vanadium dioxide $\left(\mathrm{VO}_{2}\right)$ being widely studied due to the near room temperature insulator-to-metal transition temperature. Much of the effort focused on controlling the solar properties of glazings[1], altering the transition temperature by various means (including doping and strain)[2-4], as well as some work on modulating the infrared emission.[5-7]

Here we will discuss the various measurement techniques required to accurately determine the optical / thermal response of such systems. The measurement techniques discussed apply to both switchable and non-switchable emissive surfaces.

Thus by determining the emissivity of a surface at various temperatures the integrated thermal power radiated from a surface can then be calculated via Stefan-Boltzmann law $P_{\text {out }}=\varepsilon \sigma T^{4}$.

\section{EMISSIVITY MEASUREMENT TECHNIQUES}

As there are a number of different methods available to characterise the thermal emission from a surface we will begin by discussing these various techniques. 


\subsection{Calorimetric}

Often considered the gold standard in thermal emission characterization [8], but unfortunately it can be particularly time consuming and is not available in many labs. There is still a great need for comparisons between calorimetric and spectral based emissivity calculations. However, in this paper we will be concentrating on non-calorimetric measurements.

\subsection{Emissometers}

Broadband emissometers $[9,10]$ measure integrated reflectance of a black body using a thermopile. This approach typically has the sample itself held at room temperature, while the reflectance of a heated cavity around $80^{\circ} \mathrm{C}$ is measured with a thermopile. The blackbody spectral distribution at $80^{\circ} \mathrm{C}$ rather than $25^{\circ} \mathrm{C}$ can cause a shift in attained emittance when samples have sharp spectral features, as is often the case for radiative cooling. There are various embodiments of such systems, but typically for a specular surface the near-normal incidence emittance is obtained, while for a diffuse sample a reasonable approximation of the hemispherical-to-normal emittance is measured, which is the inverse of the collection angles measured with an integrating sphere reflectance measurement (which is a normal-tohemispherical measurement).

\subsection{Spectral Reflectance}

FTIR - Fourier transform infrared spectrometers with appropriate reflectance accessories allow for spectral measurements across the required spectral range, however care must be taken to ensure appropriate well-characterised reference materials are utilised in the calibration. Often here the measurements are limited to room temperature, but we have overcome this limitation with the use of a temperature-controlled stage allowing reflectance measurements across a wide range of temperatures. Ideally measurement would be taken in a hemispherical-to-hemispherical configuration.

\subsection{Infrared Ellipsometry}

The use of ellipsometry within the infrared spectral range has added benefits for the characterisation of specular surfaces. We have retro-fitted two stepper controlled infrared wire grid polarisers to our FTIR spectrometer thus allowing us to perform temperature-dependent multi-angle ellipsometry across the infrared range. A limitation is that the samples must be specular in nature.

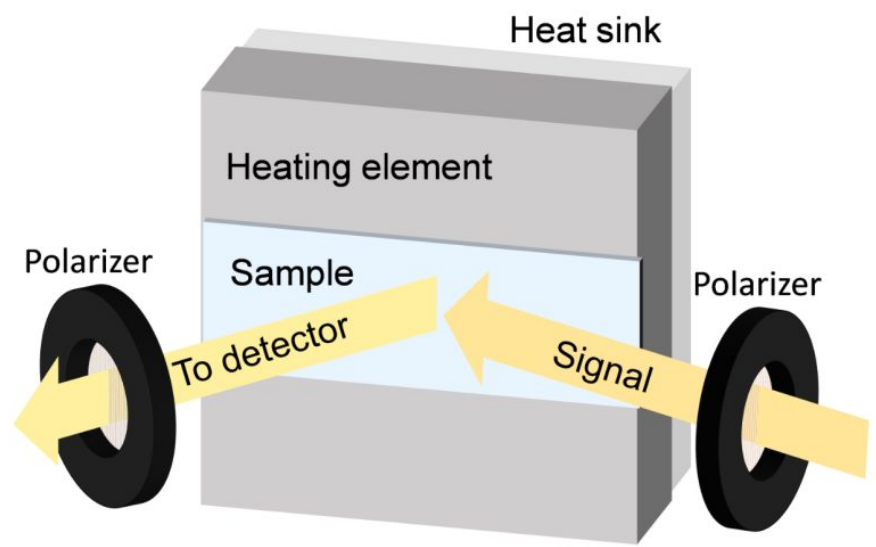

Figure 1: FTIR equipped with temperature stage, and polarisers allowing it to function as an ellipsometer.

Ellipsometry measures the complex reflection ratios of $\frac{r_{p}}{r_{s}}=\tan (\psi) e^{i \Delta}$, where $\tan (\psi)$ is the amplitude ratio, and $\Delta$ is the phase shift upon reflectance. By measuring the reflected intensity at a range of polarizer and analyzer angles a simple fitting routine can be used to determine the ellipsometric parameters. 


\subsection{Thermal Emission measurement}

Utilising a calibrated infrared thermopile and a temperature-controlled stage it is possible to determine the emittance of a surface. Many of the infrared thermopile based thermometers are limited to the $7-14 \mu \mathrm{m}$ wavelength range which is conveniently matches the spectral range of the "sky window", allowing for temperature-dependent sky window emittance, $\varepsilon_{\text {skywindow }}(T)$, to be determined. Locating the infrared detector on a goniometer allows for the emittance to be measured across a range of emission angles as well as temperatures. Computer control allows for sweeping through either sample stage temperature or emission angles, or a scan across a range of angles throughout a series of temperatures allowing full mapping of the thermal emission from a surface.

It is important to take into account the sample temperature and reflected temperatures at each angle in order to accurately determine the emittance of the surface being tested. Using an infrared sensor that returns a temperature measured in Kelvin we can use Equation 1 to determine the emittance of the sample at each angle $\varepsilon(\theta)$ and temperature.

$$
\varepsilon=\frac{T_{I R}{ }^{4}-T_{\text {Reflected }}{ }^{4}}{T_{\text {Sample }}{ }^{4}-T_{\text {Reflected }}{ }^{4}}
$$

The results presented here were collected with a CALEX PMU201 PyroMiniUSB infrared thermometer with 20:1 optics which leads to a normal incidence spot size of approximately $10 \mathrm{~mm}$, and thus they are a measure of $\varepsilon_{\text {skywindow }}(T, \theta)$ due to the spectral response of the detector. If emissivity measurements across other wavelength ranges are desired it is a simple matter of swapping the infrared sensor to one with a different spectral response.

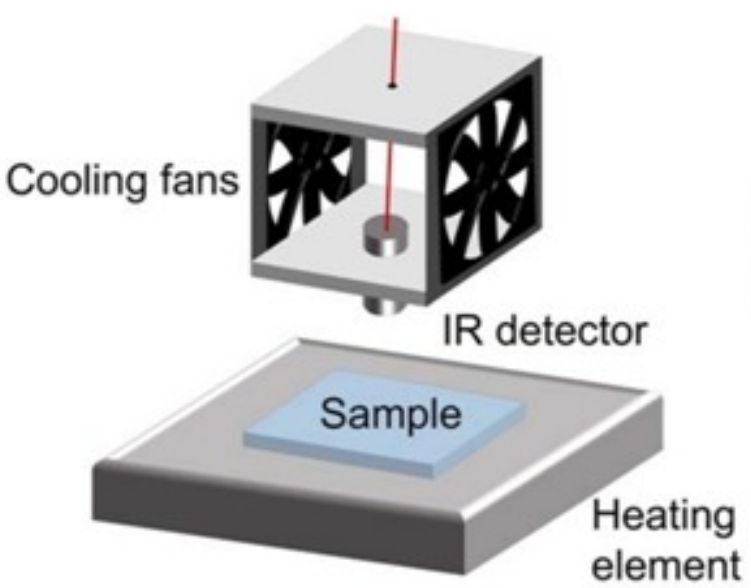

Figure 2: Schematic of the emission based emissometer.

\section{SAMPLE FABRICATION}

Our $\mathrm{VO}_{2}$ surfaces are produced via DC magnetron sputtering of metallic vanadium followed by an oxidation process. Both glass and silicon substrates are first ultrasonically cleaned in acetone followed by ethanol for 10 minutes each followed by rising in de-ionised water, and blown dry with compressed nitrogen. We first detail the fabrication methods for the metallic precursor for both planar and nanostructured $\mathrm{VO}_{2}$ followed by details of the annealing process. 


\subsection{Planar $\mathrm{VO}_{2}$}

Metallic vanadium thin films of 50nm thickness are sputtered from a 2" $99.999 \%$ purity vanadium target in a $2.7 \mathrm{mTorr}$ argon atmosphere at room temperature with a deposition current of $0.2 \mathrm{~A}$, in a cryo-pumped vacuum chamber with base pressure of $1 \times 10^{-6}$ Torr, using a quartz crystal thickness monitor to ensure thickness repeatability. Thicknesses are calibrated via ellipsometry using a Woollam V-VASE ellipsometer prior to oxidation.

\section{2 'Nano-Fin' $\mathrm{VO}_{2}$}

\subsubsection{Production of precursor structured V-Al}

The 'Nano-Fin' structured V-Al was produced by similar technique demonstrated in our previous work [11]. Specifically they were DC magnetron co-sputtered in a turbo-pumped vacuum chamber with base pressure better than $2.0 \times 10^{-5}$ Torr. Vanadium (99.999\%) and aluminum (99.95\%) targets with diameters of $50 \mathrm{~mm}$ were used and were approximately $130 \mathrm{~mm}$ vertically from the stage. During sputtering the pressure in the chamber was maintained with argon at $2.7 \mathrm{mTorr}$. Deposition currents of $0.25 \mathrm{~A}$ for vanadium and $0.3 \mathrm{~A}$ for aluminium were used. Samples were deposited for various durations of 20-60 minutes (equating to thicknesses ranging from 250 to $1200 \mathrm{~nm}$ ) to determine optimal thicknesses and tunability of thermal emission.

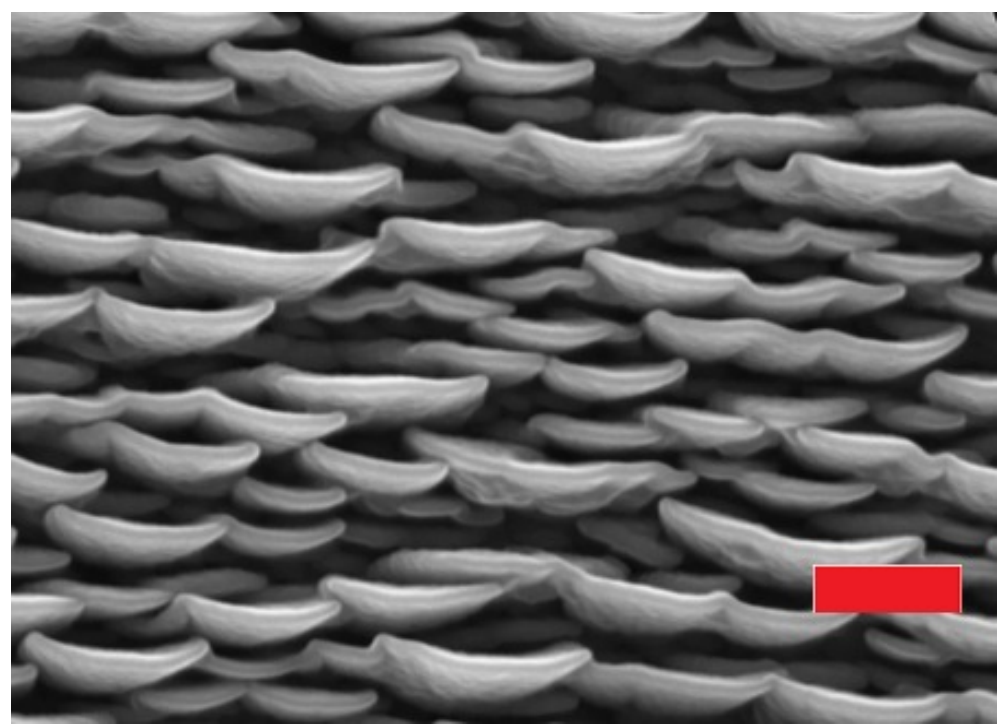

Figure 3: SEM image of the 'Nano-Fin' structured V-Al alloy. (200nm Scale bar)

\subsubsection{Formation of structured Vanadium}

The nanostructured $\mathrm{V}$ films was produced by selectively etching the $\mathrm{V}-\mathrm{Al}$ 'Nano-Fin' precursor alloy in $1 \mathrm{M} \mathrm{NaOH}$ for approximately 15 minutes, rinsed with deionised water and dried with nitrogen.

\section{3 $\mathrm{VO}_{2}$ Oxidation from metallic precursor}

Each of the samples was oxidized in an annealing chamber with in-situ optical monitoring, such that the oxidation process could be monitored. The chamber is first evacuated with a scroll pump with the pressure controlled via a computer controlled flow controller introducing air to a stable pressure of 0.3 Torr. Once pressure was stable the sample temperature is increased to a preset temperature and maintained for the duration of the oxidation process. In situ reflection measurements were taken by an Ocean Optics USB2000 every minute to observe the oxidation process. For the reflectance measurements a $5 \mathrm{~mm}$ diameter collimating and $25 \mathrm{~mm}$ diameter collection lenses (Ocean Optics 84-UV25 ) are used, leading to a $10 \mathrm{~mm}$ diameter sampling area. The light source and detector were each positioned at $15^{\circ}$ 
either side of normal incidence to the sample. Typical annealing spectra are shown in Figure 4. By monitoring the annealing process we can easily vary the oxidation conditions and terminate the processing at the appropriate time.

a)
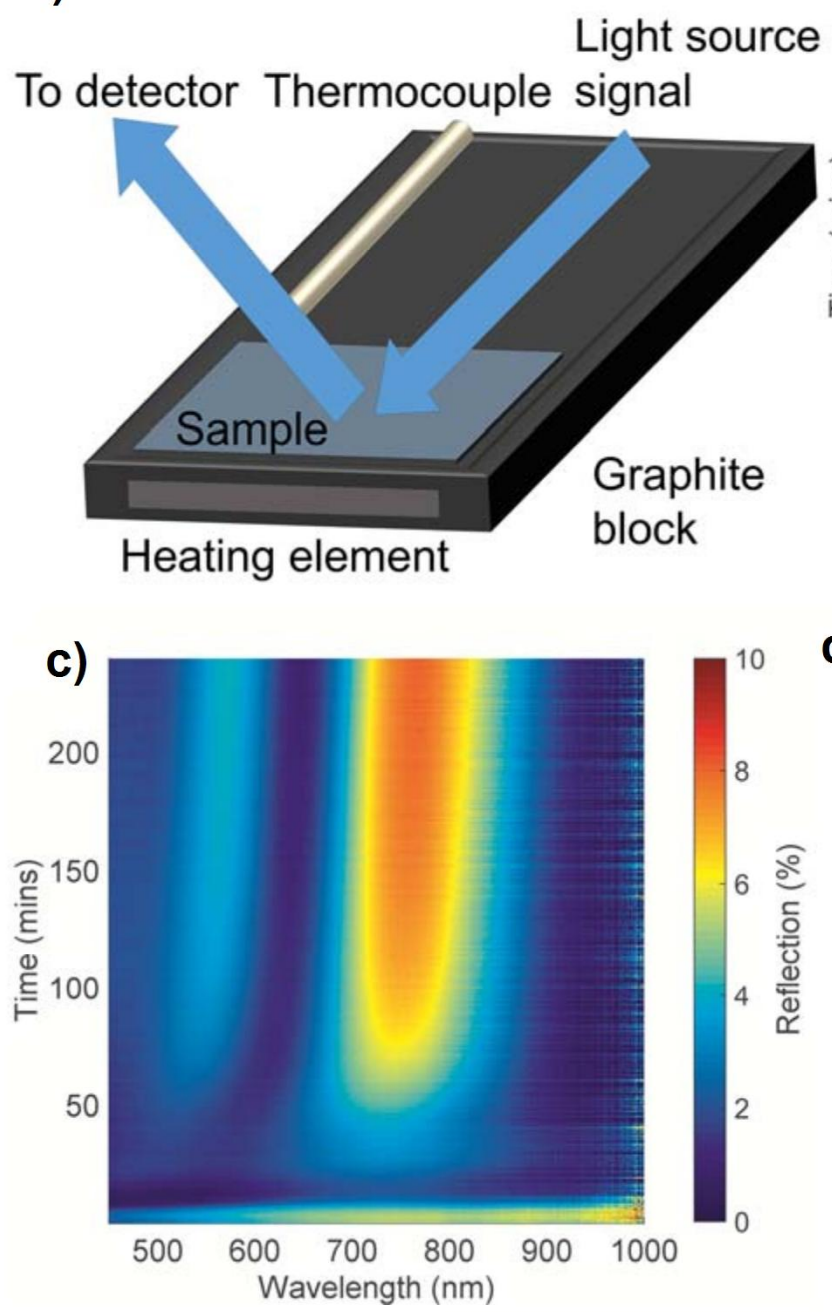

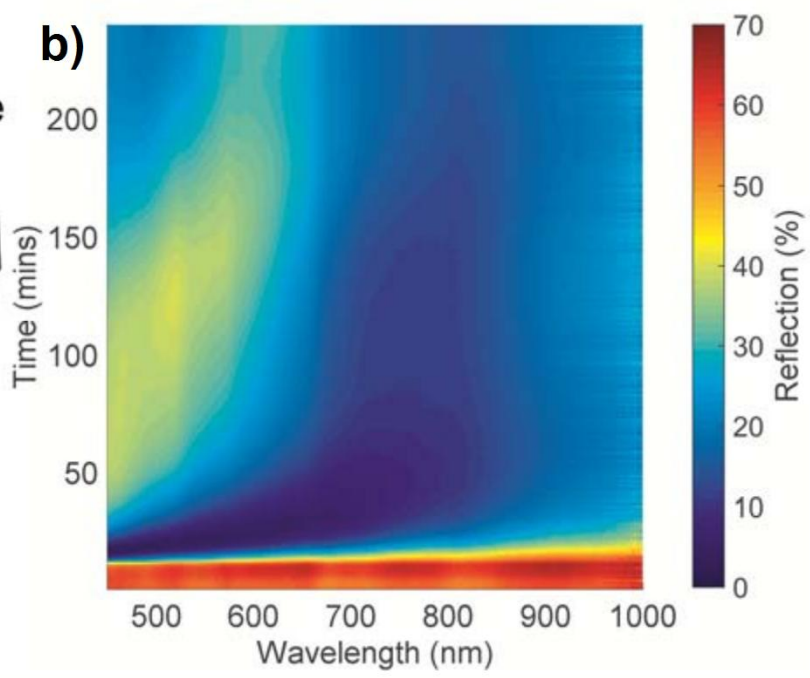

d)



Figure 4: Experimental set-up of the annealing chamber for in situ monitoring of the oxidation process via reflection measurements. A graphite stage with embedded heater and thermocouple were used to control the sample temperature. A fiber spectrometer was used to observe the change in reflection. b) In-situ reflection measurements on a 50nm thin film vanadium on a silicon substrate at $450^{\circ} \mathrm{C}, \mathrm{c}$ ) In situ reflection measurements on nanostructured thin film vanadium-aluminium deposited for 30 minutes. d) In-situ reflection measurements on thin film vanadium on glass annealed at $550^{\circ} \mathrm{C}$.

$\mathrm{V}_{2} \mathrm{O}_{5}$ is the most stable vanadium oxide if annealed at atmospheric pressure, therefore to oxidize to $\mathrm{VO}_{2}$ a lower pressure of approximately 0.1 to 1 Torr of air of must be used. A range of pressures and oxidation temperatures were investigated, we found the most repeatable films with good switching properties were attained with atmospheric pressure of 0.3Torr and a temperature between $450^{\circ} \mathrm{C}\left(250\right.$ minutes) and $550^{\circ} \mathrm{C}(20$ minutes $)$. At $450^{\circ} \mathrm{C}$, annealing time up to 24 hours was tested showing stable oxidation conditions had been achieved 


\section{OPTICAL CHARACTERISATION}

After sample fabrication samples are first measured on a Wollam V-Vase Ellipsometer with a custom in-air temperature stage allowing determination of the optical properties at room temperature and heated to $90^{\circ} \mathrm{C}$. Planar thin films of $\mathrm{VO}_{2}$ were used to fit an oscillator model consisting of a combination of Tauc-Lorentz, and Lorentz oscillators for the room temperature semi-conducting phase, with the high temperature phase consisting of Tauc-Lorentz, Lorentz and Drude oscillators. A biaxial effective medium model [11] was applied to the nanostructured $\mathrm{VO}_{2}$.

The thin films were heated with ellipsometry used to observe the insulator to metal transition at multiple wavelengths ranging from UV/Vis - IR. The hysteresis of psi plots differ greatly between thin film $\mathrm{VO}_{2}$ and nanostructured $\mathrm{VO}_{2}$ (Figure 5). An increase in psi is observed for the planar thin films, as expect due to the Drude response of the metallic state, whereas the opposite effect occurs for the nanostructured thin films. This phenomena is due to the nanostructures once they transition from insulator to metal. A plasmonic response caused by the nano-fins results in absorption of wavelengths in the infrared range. Thus by tuning the thickness of the nanostructured thin films, the emissivity and plasmonic resonance can be shifted to increase the change in emissivity. An optical model was produced to fit the ellipsometric data so that the optimal thickness and parameters could be determined. Data acquired on $\mathrm{VO}_{2}$ at room temperature and after the transition temperature were used to fit the data of the thin film $\mathrm{VO}_{2}$ within the solar and infrared ranges. The optical data assessed by the ellipsometers were combined and a basic optical model fit to 300-3500 $\mathrm{nm}$ to ensure our existing [2] $\mathrm{VO}_{2}$ optical model was valid for these samples. The IR section of the ellipsometric data was then appended to the fitted UV/Vis section of the data and oscillators were added to fit the data in the IR. Once an adequate fit was found for the thin film $\mathrm{VO}_{2}$, the nanostructured thin films were then fitted. Due to the complexity of the structures the optical model must also simulate the nanostructure. A biaxial effective medium approximation was used to simulate the anisotropic nature of these films in conjunction with a gradient graded layer since the nano-fins taper as they grow. Thicknesses of the nanostructured thin films were estimated with cross-section SEM and used as initial guesses for the fitting software. The resultant model was then used to simulate potential samples with similar structures to determine the optimal thicknesses.

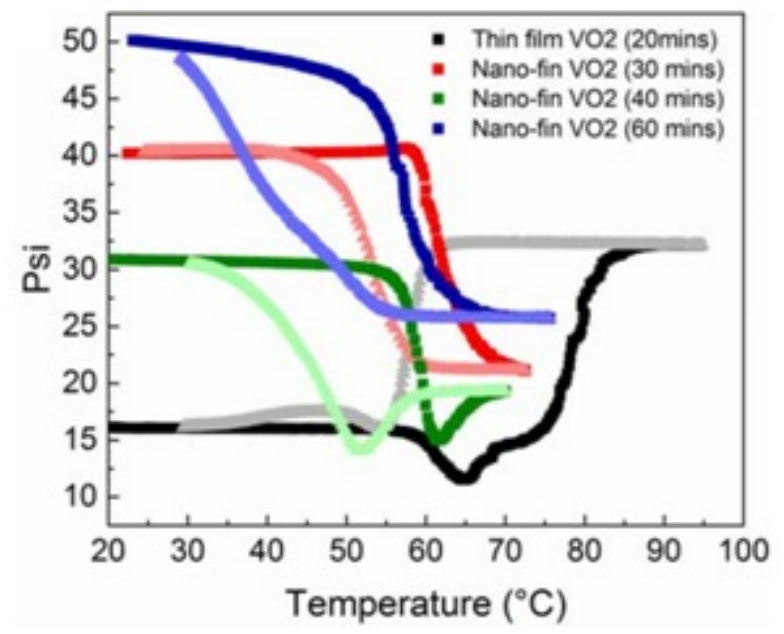

Figure 5: Hysterisis of the VO2 films as measured by ellipsomerty (2500nm) the lighter colour indicates cooling 


\subsection{Temperature Dependent Emissivity (via thermal emission measurement)}

By varying the morphology from planar films to nanostructures of various thickness we are able to fabricate samples with various thermal emmitance responses. Planar thin films of $\mathrm{VO}_{2}$ show the typical response (from high emittance to low emittance upon heating to the metallic state), whereas our structured films show different responses including lowto-high and low-high-low transitions.
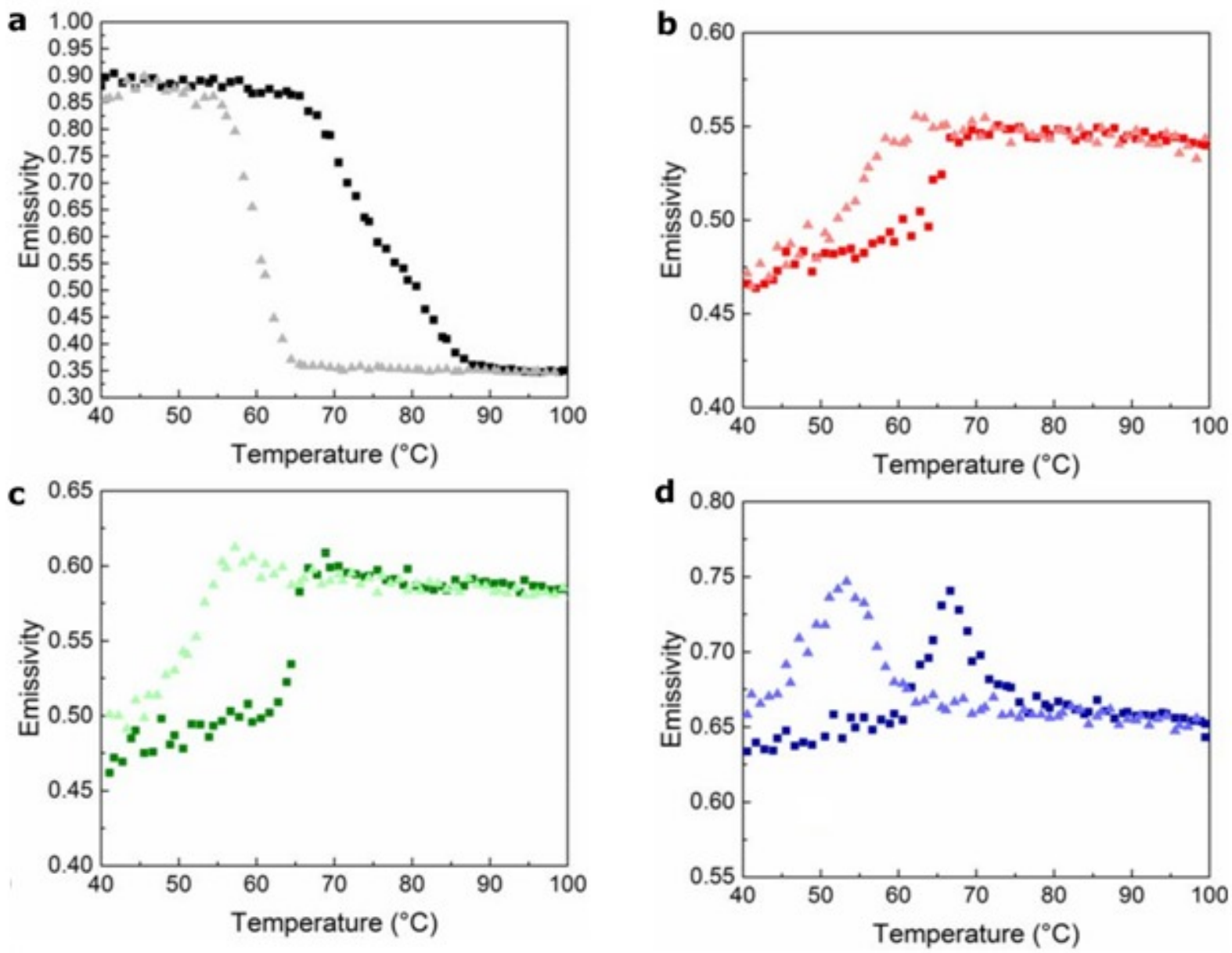

Figure 6: Normal incidence temperature dependent emissivity of (a) planar $\mathrm{VO}_{2},(b, c, d)$ various thickness nanostructured $\mathrm{VO}_{2}$ samples, showing some of the possible emittance profiles. 


\subsection{Temperature dependent angular emissivity (via thermal emission measurement)}


Figure 7: Angular dependent Emissivity E(theta), during heating from 35 to $80^{\circ} \mathrm{C}$ (left) and cooling from $80^{\circ}$ back to $35^{\circ} \mathrm{C}$. (right)

An interesting variation in angular emissivity is seen for the smooth thin film samples upon the transition from insulator to metallic state (Figure 7). In the hot(metallic) state less angular variation is observed than that of room temperature. It is often not necessary to undertake a full matrix scan as in Figure 7, with a normal incidence temperature (Figure 6), and angular (Figure 9) scans at both hot and cold states often sufficient for routine measurements.


Figure 8:(left) SEM image of $100 \mathrm{~nm}$ thick Planar $\mathrm{VO}_{2}$, (right) typical nanostructured $\mathrm{VO}_{2}$ thin film on glass. (Scale bar 200nm)

We now turn to the possibilities arising from the distinct oriented nature of the nanostructured $\mathrm{VO}_{2}$ films. These spontaneously formed features have been previously used to produced refractory polarisers in the visible range [11], which can be exploited here to further alter the thermal emission from a surface. Examples of the variations in angular emission in each place are shown in Figure 9. In this case at room temperature the emissivity is relatively isotropic, while once the structure is heated not only do we observe an increase in emittance, we also see a separation in the two planes of incidence due the anisosotropy of the structure Figure 8. We are currently in the process of optimisation of the structures for maximum emissivity anisotropy. 




Figure 9: Angular dependent emissivity, showing the anisotropic response at high temperature of a typical 800 $\mathrm{nm}$ film.

This measurement technique is particularly useful for materials such as $\mathrm{VO}_{2}$ with significant variation in emissivity across a range of temperature, while at the same time beneficial for more measuring typical materials which a measurement of the angular emissivity is of interest with respect to various coating and texturing, measured at the actual operating temperature.

\section{DISCUSSION AND CONCLUSIONS}

We have demonstrated a method of determining angular dependent emissivity, $\varepsilon(\theta)$, which is applicable to both specular and diffuse samples without the need for external correction factors or integrating spheres. In order to calculate thermal balances of systems it is essential to account for the quantities of radiation emitted and absorbed at each angle. In the case of maximising emittance, one desires high emittance at all emission angles. The measurement methods here allow for simple evaluation of the angular effects arising from various applied coatings and textures.

In order to evaluate the flexibility of the system we produced $\mathrm{VO}_{2}$ thin films and nanostructures which exhibit both positive and negative differential emission. Coatings which can increase their emissivity as temperature increases are of particular interest for radiatively cooled self regulating emitting surfaces. Formation of appropriately sized features in vanadium which can be oxidised to $\mathrm{VO}_{2}$ opens up a new method for plasmonically controlling radiative transfer from a $\mathrm{VO}_{2}$ surface.

\section{ACKNOWLEDGEMENTS}

This research is supported by an Australian Research Council Discovery Project Grant DP140102003. 


\section{REFERENCES}

1. Mlyuka, N.R., G.A. Niklasson, and C.G. Granqvist, Thermochromic VO2-based multilayer films with enhanced luminous transmittance and solar modulation. physica status solidi (a), 2009. 206(9): p. 2155-2160.

2. Gentle, A., A. Maaroof, and G. Smith, Nanograin VO2 in the metal phase: a plasmonic system with falling dc resistivity as temperature rises. Nanotechnology, 2006. 18(2): p. 025202.

3. Gentle, A.R., G.B. Smith, and A.I. Maaroof, Frequency and percolation dependence of the observed phase transition in nanostructured and doped VO2 thin films. Journal of Nanophotonics, 2009. 3(1): p. 031505.

4. Mlyuka, N., G.A. Niklasson, and C.-G. Granqvist, Mg doping of thermochromic VO 2 films enhances the optical transmittance and decreases the metal-insulator transition temperature. Applied physics letters, 2009. 95(17): p. 171909.

5. Leahu, G., et al., Anomalous optical switching and thermal hysteresis during semiconductormetal phase transition of VO2 films on Si substrate. Applied Physics Letters, 2013. 103(23): p. 231114.

6. Hendaoui, A., et al., Highly tunable-emittance radiator based on semiconductor-metal transition of VO2 thin films. Applied Physics Letters, 2013. 102(6): p. 061107.

7. Kats, M.A., et al., Vanadium dioxide as a natural disordered metamaterial: perfect thermal emission and large broadband negative differential thermal emittance. Physical Review X, 2013. 3(4): p. 041004.

8. Millard, J.P. and E.R. Streed, A Comparison of Infrared-Emittance Measurements and Measurement Techniques. Applied Optics, 1969. 8(7): p. 1485-1492.

9. ASTM C1731-15, Standard Test Method for Determination of Emittance of Materials Near Room Temperature Using Portable Emissometers. ASTM International, West Conshohocken, PA, 2015.

10. Bevans, J., E. Luedke, and K. Nelson, A device for the rapid measurement of total emittance. Journal of Spacecraft and Rockets, 1966. 3(5): p. 758-760.

11. Tai, M.C., et al., Spontaneous growth of polarizing refractory metal 'Nano-Fins'. Nanotechnology, 2018. 